\title{
Clarification of Unsteady Fluid Forces Acting on Limbs in Swimming Using an Underwater Robot Arm
}

\author{
(2nd Report, Modeling of Fluid Force Using \\ Experimental Results)*
}

\author{
Motomu NAKASHIMA** and Akemi TAKAHASHI*** \\ ${ }^{* *}$ Graduate School of Science and Engineering, Tokyo Institute of Technology \\ 2-12-2 Oookayama, Meguro-ku, Tokyo 152-8552, Japan \\ E-mail: motomu@mech.titech.ac.jp \\ ***Graduate School of Information Science and Engineering, Tokyo Institute of Technology
}

\begin{abstract}
The objective of this study was to clarify the unsteady characteristics of the fluid force acting on limbs during swimming. For this objective, an underwater robot arm, which has five degrees-of-freedom in order to perform the various complicated limb motions during swimming, was developed. In the previous study, an experiment to measure the unsteady fluid force was conducted for four swimming strokes of the upper and lower limbs. In this paper, the unsteady fluid force model was firstly formulated. Second, the simulation of experimental conditions was conducted. Two fluid force coefficients, which are the parameters in the fluid force model, were identified using optimizing calculation, so that the discrepancies of the forces and moments between the experiment and simulation were minimized. In addition, fluid force models which are dependant only on the limbs' shapes were determined. Good agreement between the experiment and simulation with the determined fluid force model indicated the validity of the determined model. The identified fluid model will be useful for mechanical analyses of various swimming motions in future studies.
\end{abstract}

Key words: Swimming, Sport Engineering, Fluid Force, Bio-Fluid Mechanics, Underwater Robot Arm

\section{Introduction}

Although the thrust produced by four limbs is known to be important in swimming, the fluid force acting on the limbs has not been sufficiently clarified. The objective of this study was to clarify the unsteady fluid forces acting on the limbs using an underwater robot arm which has five degrees-of-freedom (DOF) and can perform swimming motions similar to the human one. In the preceding report ${ }^{(1)}$, a robot arm was developed and an experiment to measure the unsteady fluid forces was conducted. However, it could not be said that those characteristics were fully clarified if the fluid forces were only measured experimentally. Also in the previous experiments to measure the steady fluid forces acting on hand replicas, it was generally accepted to examine the measured data using drag/lift coefficients ${ }^{(2)(3)(4)}$. This is nothing less than the modeling of the fluid forces, since the fluid forces in those studies were formulated to be proportional to the density, reference area, and the square of the velocity, and then the coefficients of the formulation were determined. Therefore, it is

Received 16 Jan., 2012 (No. T2-09-0551) Japanese Original : Trans. Jpn. Soc. Mech. Eng., Vol.76, No.761, B (2010), pp.66-75 (Received 15 June, 2009) [DOI: 10.1299/jfst.7.114]

Copyright (C) 2012 by JSME 
also necessary to model the unsteady forces for clarification by assuming a certain formulation and determining the parameters in the formulation. If such a model is established, it will be versatile and useful for various mechanical analyses in swimming. Therefore, in this study, the unsteady fluid forces acting on the four limbs during swimming were modeled based on the experimental results in the preceding report. The formulation of the fluid force model was carried out the same as the swimming human simulation model SWUM $^{(5)(6)(7)}$, which has already been developed by the authors. In this formulation, the fluid force, except for buoyancy, is represented as the sum of two components. One component is proportional to the square of the velocity and represents the drag/lift force the same as the previous studies. The other component is proportional to the acceleration and represents the effect of the inertial force due to the added mass of the fluid. It has already been confirmed that this formulation is also valid for unsteady fluid forces in the previous report $^{(5)}$. However, it has not been sufficiently examined yet for more complicated swimming motions since the examination of the previous report was confined to the experiment in which a 1 DOF motion was performed by a simple bar-shaped limb replica. In this paper, the outline of the experiment to measure the fluid forces in the previous report is explained briefly, followed by the description of the modeling method of the fluid force. The parameters in the fluid force model (fluid force coefficients) are identified so that the results of the simulation using the model become consistent with those of the experiment as closely as possible. Finally, the results of the simulation using the identified fluid force model are shown and the validity of the present fluid force model is examined. The novel point of this paper was, in short, to construct the fluid force model which can reproduce, with a small amount of computation, the unsteady fluid force acting on the limbs during swimming measured by means of an underwater robot arm. No such model has existed to date.

\section{Outline of the experiment to measure fluid forces}

\subsection{Underwater robot arm}

The underwater robot arm which was developed for the experiment to measure fluid forces is shown in Fig. 1. The robot arm consists of the trunk, shoulder, upper arm, forearm and hand (here the robot arm is described as the upper limb, although it can perform motions of both the upper and lower limbs). It has 3 and 2 DOF at the shoulder and elbow, respectively. The shoulder part performs the rotation on the A-axis (Flapping) which is fixed to the trunk part. The upper arm part performs 2 DOF rotations, which are the rotation on the B-axis (Rowing) which is fixed to the shoulder part, and the twisting rotation. The forearm part performs the flexion/extension and pronation/supination of the elbow. The swimming motion is reproduced by the combination of these 5 DOF. Tri-axis moment sensors using strain gauges were installed in the root parts of the upper arm, forearm and hand, and they

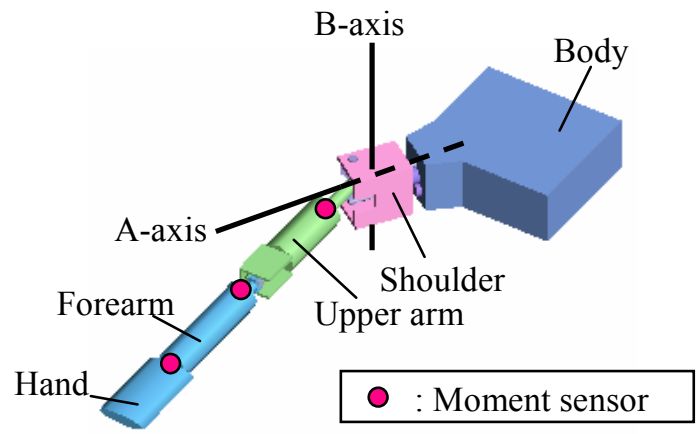

(a) Schematic view

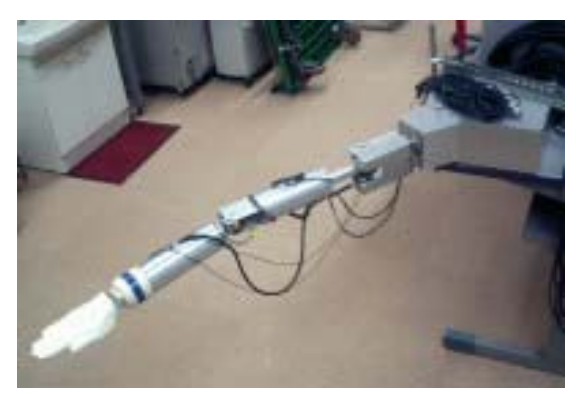

(b) Photograph

Fig. 1 Developed robot arm 


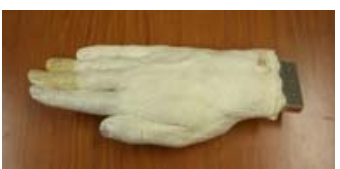

(a) Right hand

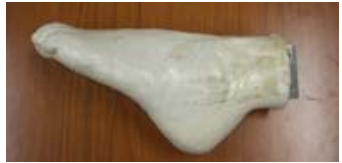

(c) Plantarflexed foot

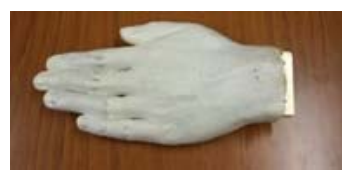

(b) Left hand

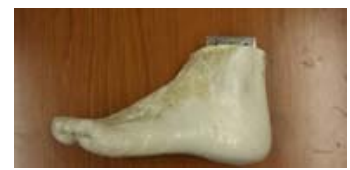

(d) Dorsiflexed foot

Fig. 2 Photographs of replicas

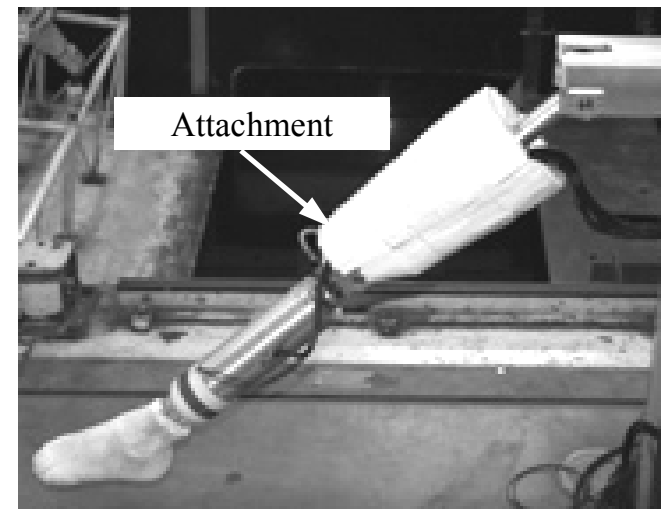

Fig. 3 Attachment to simulate thigh

measured the moments at the places which correspond to the shoulder, elbow, and wrist of a human. A dynamometer was installed at the top of the trunk, by which the load acting on the whole robot arm was measured. The hand part was designed to be exchangeable. The hand and foot replicas were mounted for performing the motions of the upper and lower limbs, respectively. Photographs of the replicas are shown in Fig. 2. Four hand/foot replicas were made: the right hand for the crawl, breaststroke and butterfly, the left hand for the backstroke, the plantarflexed (ankle joint is extended) foot for the crawl, backstroke and butterfly, and the dorsiflexed (ankle joint is flexed) foot for the breaststroke. When the lower limb motion was performed, an attachment, which imitated the thigh and was made of urethane foam, was installed at the thigh part so that the ratio of diameters of the thigh (corresponding to the upper arm) and shank (corresponding to the forearm) became the same as that of the average of Japanese adults ${ }^{(8)}$. The appearance of the attachment simulating the thigh is shown in Fig. 3. The swimming motion is arbitrarily programmable due to the computer control.

\subsection{Contents of experiment}

The motions of the upper and lower limbs for the four strokes (crawl, back, breast and butterfly) were performed in the experiment. For the upper limb of the crawl, two kinds of strokes were performed, that is, the 'S-shaped stroke' in which the hand stroke became an S-shaped curve, and the 'I-shaped stroke' in which the hand stroke became a relatively straight line. As the experimental condition for each case, the joint angles for one cycle, stroke cycle, mounting height of the robot arm, and the flow velocity were given. The joint angles were determined based on movies of an actual athlete swimmer swimming as a model. With respect to the relationship between the flow velocities and stroke cycles, the flow velocities were determined so that the normalized stroke length, which represented the advancing distance in one stroke cycle divided by the swimmer's stature, became as 
consistent as possible with those in the movie of the model swimming. An experiment to identify the inertial characteristics of the robot arm was conducted on land for the simulation reproducing the experiment. Full details of the above contents are available in the previous report ${ }^{(1)}$.

\section{Modeling method of Fluid Forces}

\subsection{Formulation of the fluid force model}

The robot arm was modeled as a series of simple truncated elliptic cones in the present study. The fluid forces acting on the truncated elliptic cones which represent parts of the robot arm in the present fluid force model are shown in Fig. 4. The fluid forces are assumed to be representable as the sum of the inertial force due to the added mass of the fluid, $\boldsymbol{F}_{a}$, the drag force normal to the longitudinal axis of the truncated elliptic cone, $\boldsymbol{F}_{n}$, the drag force tangential to that, $\boldsymbol{F}_{t}$, and buoyancy, $\boldsymbol{F}_{b}$. One truncated elliptic cone is divided into the thin elliptic plates along the longitudinal direction, as shown in Fig. 4(a). The fluid forces except buoyancy are assumed to act on the centers of the elliptic plates. This assumption was made as the first step of the modeling although these forces do not always actually act on just the centers of the elliptic plates. Buoyancy is assumed to act on the tiny quadrangles into which the edges of the thin elliptic plates are again divided into in the circumferential direction, as shown in Fig. 4(b). An example of an elliptic plate into which the truncated elliptic cone is divided is shown in Fig. 5. The symbols $\boldsymbol{e}_{1}, \boldsymbol{e}_{2}, r_{1}, r_{2}, \boldsymbol{a}_{n}, \boldsymbol{v}_{n}, \boldsymbol{v}_{t}$ and $d l$ respectively represent the unit vectors in the directions of the ellipse's minor and major axes, the half-lengths of the minor and major axes, the absolute acceleration vector of the center of the elliptic plate, the absolute velocity vectors in the normal (perpendicular to the longitudinal axis of the truncated elliptic cone) and tangential (parallel to the longitudinal axis) directions, and the thickness of the plate.

The inertial force due to the added mass of the fluid for an elliptic plate, $\boldsymbol{F}_{a}$, is assumed to be given by:

$$
\boldsymbol{F}_{a}=-C_{a} \rho \varepsilon_{f} d l \pi\left\{r_{2}^{2}\left(\boldsymbol{a}_{n} \cdot \boldsymbol{e}_{1}\right) \boldsymbol{e}_{1}+r_{1}^{2}\left(\boldsymbol{a}_{n} \cdot \boldsymbol{e}_{2}\right) \boldsymbol{e}_{2}\right\}
$$

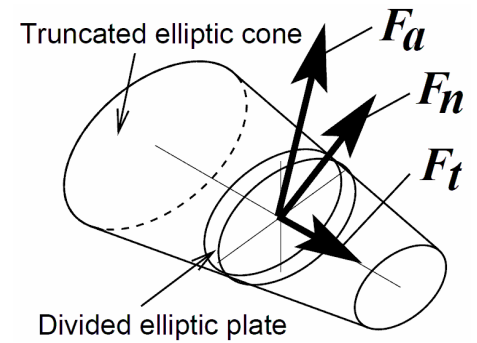

(a)

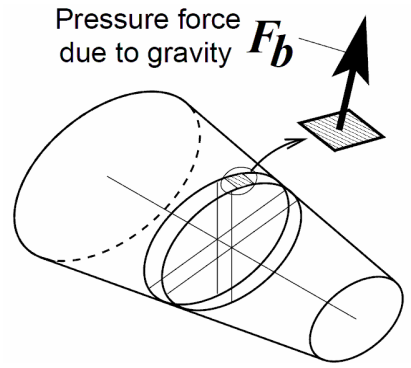

(b)

Fig. 4 Acting point of fluid force in the model

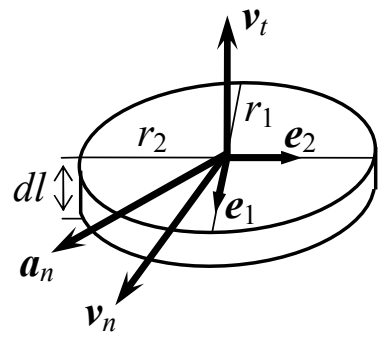

Fig. 5 Elliptic plate 
where $C_{a}$ is the coefficient used to correct the fluid forces for a three-dimensional real fluid. The coefficient, $\varepsilon_{f}$, defined later, represents the 'submerged fraction' of the plate. In Eq. (1), the acceleration, $\boldsymbol{a}_{n}$, is decomposed into the components of $\boldsymbol{e}_{1}$ and $\boldsymbol{e}_{2}$, the inertial force due to the added mass for each direction is calculated, and these forces are again composed. Note that $C_{a}$ is a constant value and $C_{a}=1$ holds in a two-dimensional ideal fluid. That is, if $C_{a}=1$ and $\varepsilon_{f}=1$, Eq. (1) is strictly consistent with the theoretical expression of the inertial force due to added mass acting on an arbitrary elliptic cylinder in a two-dimensional ideal fluid.

The drag force in the normal direction, $\boldsymbol{F}_{n}$, is assumed to be given by:

$$
\boldsymbol{F}_{n}=-C_{n} \rho \varepsilon_{f} d l\left\{r_{2}\left(\frac{r_{2}}{r_{1}}\right)^{p}\left|\boldsymbol{v}_{n}\right|\left(\boldsymbol{v}_{n} \cdot \boldsymbol{e}_{1}\right) \boldsymbol{e}_{1}+r_{1}\left(\frac{r_{1}}{r_{2}}\right)^{p}\left|\boldsymbol{v}_{n}\right|\left(\boldsymbol{v}_{n} \cdot \boldsymbol{e}_{2}\right) \boldsymbol{e}_{2}\right\}
$$

where $C_{n}$ represents the drag coefficient for the normal direction. In Eq (2), the velocity, $\boldsymbol{v}_{n}$, is decomposed into the components of $\boldsymbol{e}_{1}$ and $\boldsymbol{e}_{2}$, the drag force for each direction is calculated, and these forces are again composed. When $r_{1}=r_{2}, \boldsymbol{F}_{n}$ is in the direction just reverse to $\boldsymbol{v}_{n}$, and this formulation becomes the normal equation for the drag acting on a circular cylinder. When $r_{1} \neq r_{2}$, a lift force, which is perpendicular to $\boldsymbol{v}_{n}$ and the longitudinal axis of the truncated elliptic cone, is generated. This means that not only the drag force, but also the lift force is represented in Eq. (2). With respect to the multiplier $p$ in the equation, $p$ $=1.0$ is assumed since it was identified as 1.0 by the experiment in the previous report ${ }^{(2)}$. The parts of $\left(r_{2} / r_{1}\right)^{p}$ and $\left(r_{1} / r_{2}\right)^{p}$ in Eq. (2) were added to control the effect of flatness of the ellipse on the fluid force. The coefficient $C_{n}$ is assumed to be constant in this study.

The drag force in the tangential direction, $\boldsymbol{F}_{t}$, is assumed to be given by:

$$
\boldsymbol{F}_{t}=-\frac{1}{2} C_{t} \rho \varepsilon_{f} d l c\left|\boldsymbol{v}_{t}\right| \boldsymbol{v}_{t}
$$

where $C_{t}$ and $c$ represent the drag coefficient for the tangential direction and the ellipse's circumference, respectively. The coefficient $C_{t}$ is assumed to be constant, the same as $C_{n}$.

Buoyancy, $\boldsymbol{F}_{b}$, is given by:

$$
\boldsymbol{F}_{b}=-z_{b} d s g \boldsymbol{e}_{n}
$$

where $d s, \boldsymbol{e}_{n}, z_{b}$ and $g$ represent the area of the tiny quadrangle, the vector normal to the quadrangle, $z$ coordinate of the quadrangle center, and the gravitational acceleration, respectively. The water surface is assumed to be flat and $z_{b}=0$ holds at the water surface. Since buoyancy does not act above the water surface, only $\boldsymbol{F}_{b}$ acts on the quadrangle for which $z_{b}<0$ holds. Since this classification is performed for each tiny quadrangle in the simulation program, the ratio (number of quadrangles where $z_{b}<0$ holds)/(number of all quadrangles) can be calculated for each elliptic plate. In the present model, this ratio is called the 'submerged fraction,' $\varepsilon_{f}$, by which $\boldsymbol{F}_{a}, \boldsymbol{F}_{n}$ and $\boldsymbol{F}_{t}$ are multiplied.

The fluid force acting on one truncated elliptic cone is calculated by summing up the above fluid forces for all elliptic plates along the longitudinal axis of the truncated elliptic cone. The total fluid force acting on the robot arm is then calculated as the sum of the fluid forces for all the truncated elliptic cones.

The gravitational force, $\boldsymbol{F}_{g}$, and inertial force, $\boldsymbol{F}_{i}$, for one elliptic plate are given by: 


$$
\begin{aligned}
& \boldsymbol{F}_{g}=m g \boldsymbol{z} \\
& \boldsymbol{F}_{i}=-m \boldsymbol{a}
\end{aligned}
$$

where $m, \boldsymbol{z}$ and $\boldsymbol{a}$ represent the mass of the elliptic plate, the vector vertically downward, and the absolute acceleration of the elliptic plate, respectively.

\subsection{Modeling of underwater robot arm}

The simulation model of the robot arm is schematically shown in Fig. 6. In the lower limb model shown in Fig. 6(b), the heel is placed at the bottom and the toe is extended. The upper arm and forearm (thigh and shank) are represented by multiple cylinders whose radii are different, while the hand (foot) is represented by a truncated elliptic cone. The dimensions of the truncated elliptic cones were determined using the anatomical dimensions of the hand and foot replicas as follows: As shown in Fig.7(a), for the major and minor axis lengths of the trunk side of the hand replica, the hand width including the thumb and the thickness of the wrist, were used. For the major and minor axis lengths of the tip, the width from the second to the fourth fingers and the average thickness from the second to the fifth

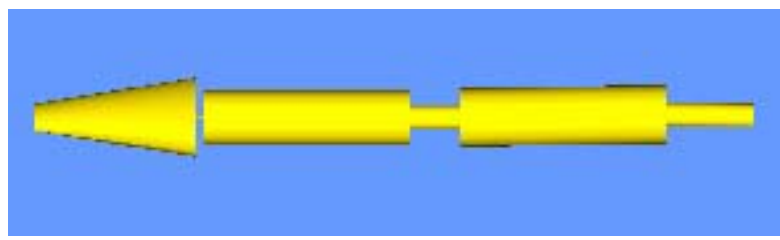

(a) Upper limb

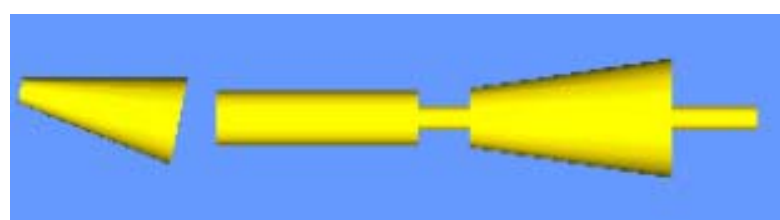

(b) Lower limbs

Fig. 6 Simulation model (schematic view)

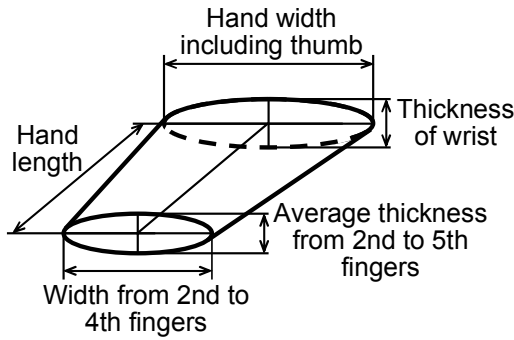

(a) Dimensions for hand

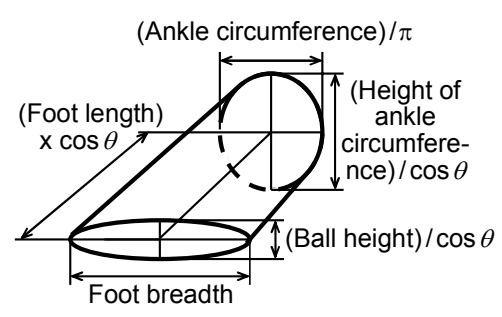

(b) Dimensions for foot

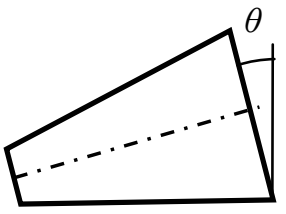

(c) Definition of angle $\theta$, which is calculated as $\theta=\sin ^{-1}((($ height of ankle circumference $)-($ ball height $) /($ foot length $))$

Fig. 7 Definitions of dimensions for hand and foot 
fingers were used. For the height of the truncated elliptic cone, the hand length was used. In order to determine the foot dimensions, ankle circumference, the height of the ankle circumference, ball height, foot length, foot breadth, and $\theta$ were used as shown in Fig.7(b). The angle, $\theta$, is defined as the angle between the end face of the truncated elliptic cone and the vertical plane when the foot bottom is parallel to the land surface in the standing position, as shown in Fig.7(c). The measured lengths of the replica were converted into the dimensions of the ellipse using $\theta$. For the center of the end face of the foot replica's trunk side, the midpoint of the lateral and medial malleoli was used. Since the influences of the wrist (ankle) part and the plate to mount the hand/foot replica to the robot arm were considered to be small, these were not modeled, shown as a gap in Fig. 6. The mass centers of the truncated elliptic cones and the inertial characteristics were identified using the results of the experiment on land.

\subsection{Implementing the method of the model and analysis conditions}

As the implementing method of the above fluid force model, the swimming human simulation software Swumsuit, which was developed by the authors ${ }^{(9)(10)}$, was employed. The dividing number of the truncated elliptic cones in the longitudinal direction and the time step were 10 and 1/144 for one cycle, respectively. As the joint angles for one cycle, the averages of measured values for five cycles in the experiment were used.

\section{Identification of Fluid Force Coefficients}

\subsection{Identification method of coefficients using optimizing calculation}

In this section, the fluid force coefficients, which are the coefficients in the fluid force model described in the previous section, are identified. Among the fluid forces $\boldsymbol{F}_{a}, \boldsymbol{F}_{n}$ and $\boldsymbol{F}_{t}$ which include the coefficients, two forces, $\boldsymbol{F}_{a}$ and $\boldsymbol{F}_{n}$, are the main components of the fluid forces acting on the four limbs. The force, $\boldsymbol{F}_{t}$, on the other hand, corresponds to the drag mainly acting on the trunk. Consequently, it is not appropriate to identify $C_{t}$ in Eq. (3) for $\boldsymbol{F}_{t}$ by the present robot arm experiment. The two coefficients, $C_{n}$ and $C_{a}$ were therefore identified in the present study, while the value identified in the previous report ${ }^{(2)}$ was used for $C_{t}$. As the optimizing method for the identification, the Downhill Simplex method, which is programmed into Swumsuit as the optimizing function, was employed. The objective function and the constraints are given by:

$$
\left[\begin{array}{l}
\text { Minimize: } f\left(C_{n}, C_{a}\right) \\
\text { Subject to: } C_{n}>0, C_{a}>0
\end{array}\right.
$$

where the objective function, $f$, is the average of weighting squared errors between the measurement in the experiment and the simulation for one cycle. The fluid force coefficients, $C_{n}$ and $C_{a}$, as the design variables, were determined so that $f$ was minimized. As the constraints, the design variables $C_{n}$ and $C_{a}$ were assumed to always be positive.

Different values of the fluid force coefficients were assumed for the hand and arm (upper arm and forearm). For the identifying procedure, the fluid force coefficients for the hand was firstly determined using the measurement results of the moment sensor at the wrist, which was subjected only to the drag on the hand. The coefficients of the arm were secondly determined using the other measurement results.

The objective function which was used to identify the fluid force coefficients of the hand part is given by:

$$
f_{\text {hand }}\left(C_{n \text { hand }}, C_{\text {a hand }}\right)=M_{X \text { wrist err }}+M_{Y \text { wrist err }}
$$




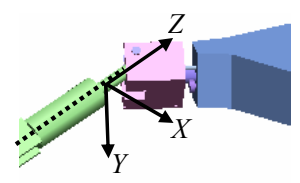

(a) Shoulder

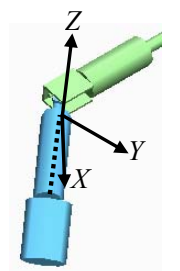

(b) Elbow

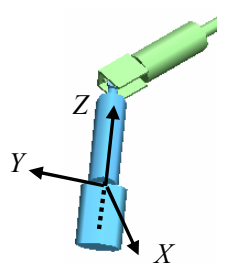

(c) Wrist

Fig. 8 Local co-ordinate system for bending moment sensors

where $X$ and $Y$ are the coordinates of the moment sensor, whose definitions are shown in Fig. 8. The symbols $M_{X \text { wrist err }}$ and $M_{Y \text { wrist err }}$ represent the sums of squared errors of $X$ and $Y$ components of the bending moments at the wrist, respectively.

The objective function which was used to identify the fluid force coefficients of the arm part is defined by:

$$
\begin{aligned}
f_{\text {arm }}\left(C_{\text {n arm }}, C_{\text {a arm }}\right)= & w_{1 s} M_{X \text { shoulder err }}+w_{1 e} M_{\text {Yelbow err }} \\
& +w_{2 s} M_{Y \text { shoulder err }}+w_{2 e} M_{X \text { elbow err }}+F_{x \text { err }}
\end{aligned}
$$

For the arm, the mean squared error of the thrust, $F_{x}$, measured by the dynamometer, which was represented as $F_{x e r r}$, was added to the objective function as well as the bending moments at the shoulder and elbow. The reason why $F_{x \text { err }}$ was added is that the thrust, which is the most important quantity in swimming, has to be estimated accurately in the present model. For the lower limb cases of the flutter kick and butterfly, $F_{x}$ was almost not generated, as described in the previous report ${ }^{(1)}$. Therefore, for these cases, the moment about the hip joint, $M_{Y}$, was used instead of $F_{x}$.

Comparing the bending moments at the shoulder and elbow in the plane formed by the upper arm and forearm, it was found that the absolute value of the moment at the shoulder tended to become larger than that at the elbow because of the difference of the lengths of the moment arm. Therefore, in order to consider equally both effects in the identification of the fluid force coefficients, a weighting procedure was conducted so that the mean squared error of the moment at the elbow became close to that at the shoulder. The weighting procedure is described as follows: In the coordinates of the moment sensors shown in Fig. 8, the $Z$ axes of the moment sensors are consistent with the center axes of the parts at which the moment sensors are mounted (that is, upper arm and forearm). Both the $Y$ axis of the shoulder and the $X$ axis of the elbow are perpendicular to the plane formed by the center axes of the upper arm and forearm, while remains are perpendicular to these two axes. Therefore, the $Y$ axis of the shoulder and the $X$ axis of the elbow are in the same direction. The same relationship holds for the $X$ axis of the shoulder and the $Y$ axis of the elbow. The weighting of the sums of squared errors of the moment was conducted for these pairs of axes in the same directions. The weight was calculated as the average of the ratio of one moment to the other at each instance for each case, and it was multiplied to the other. For example, $w_{1 s}$ in Eq. (9) is the average of $M_{Y}$ at the elbow divided by $M_{X}$ at the shoulder, while $w_{1 e}$ is its reciprocal. Similarly, $w_{2 s}$ is the average of $M_{X}$ at the elbow divided by $M_{Y}$ at the shoulder, while $w_{2 e}$ is its reciprocal.

\subsection{The results of identification and discussion}

The results of identification of the fluid force coefficients for all cases are shown in Table 1. With respect to $C_{n}$, it was found that the coefficients were within close ranges for all cases of the upper limb and for the three strokes of the lower limb, except the breaststroke. It was also found that $C_{a}$ varied relatively widely. One possible reason for this 
Table 1 Identified fluid force coefficients

\begin{tabular}{llcccc}
\hline & & \multicolumn{2}{c}{ Hand } & \multicolumn{2}{c}{ Arm } \\
\cline { 3 - 6 } & & $C_{n}$ & $C_{a}$ & $C_{n}$ & $C_{a}$ \\
\hline Upper limb & Crawl S & 0.68 & 0.00 & 2.71 & 1.15 \\
& Crawl I & 0.69 & 0.25 & 3.60 & 2.74 \\
& Breast stroke & 0.72 & 0.21 & 2.39 & 1.63 \\
& Butterfly & 0.67 & 0.22 & 3.53 & 0.95 \\
& Backstroke & 0.61 & 0.37 & 2.54 & 0.51 \\
\hline Lower limb & Crawl & 0.54 & 0.24 & 1.06 & 0.02 \\
& Backstroke & 0.51 & 0.25 & 1.04 & 0.00 \\
& Butterfly & 0.53 & 0.27 & 1.11 & 0.00 \\
& Breast & 1.88 & 0.50 & 2.30 & 2.38 \\
\hline
\end{tabular}

is that the components of $C_{a}$ were small from the first. For the versatility, an identical fluid force coefficient which is not affected by case is desirable. However, the shape of the arm for upper limbs and that of the leg for lower limbs were different from each other due to the presence/absence of the attachment to imitate the thigh. In addition to this, the shapes of the foot replicas for the breaststroke and the other three strokes were different from each other. Therefore, the unification of the coefficients for each shape was attempted by classifying them by shape. This meant to propose the versatile fluid force model whose coefficients do not depend on the motion. The procedure of the unification is as follows: First, as the index representing how much the change of the coefficients in the simulation influences on the change of the thrust, the magnitude of contribution to the thrust, $C$, was defined as:

$$
C=\left|\frac{\sum_{j=1}^{n}\left|F_{0 j}\right|-\sum_{j=1}^{n}\left|F_{1 j}\right|}{\sum_{j=1}^{n}\left|F_{0 j}\right|}\right|
$$

where $F_{0}$ and $F_{1}$ are values of $F_{x}$ before and after the change of the coefficients with a magnitude of 1.0. This value was calculated for each case. For the stroke in which the magnitude of the contribution was large, the change in the coefficient should be small in the unification. On the contrary, for the stroke in which the magnitude was small, a large change in the coefficient was acceptable. The weight was calculated as the magnitude of the contribution for each coefficient of each case, which was divided by the sum of the magnitude of contributions for all cases. The unified coefficient was finally calculated as the sum of the coefficients for all cases, which were multiplied by the respective weights. The unified coefficients are shown in Table 2.

There is a possibility of an increase in the error of the simulation against the experimental values due to the unification of the coefficients. Therefore, the effect of the unification of the coefficients on the simulated results was examined using the precision, $P$, which is defined by:

$$
P=\frac{\sum_{i=1}^{n}\left|M_{\text {simi }}-M_{\text {expi }}\right|}{n\left(M_{\max }-M_{\min }\right)}
$$


Table 2 Unified fluid force coefficients

\begin{tabular}{llllll}
\hline & & \multicolumn{2}{c}{ Hand } & \multicolumn{2}{c}{ Arm } \\
\cline { 3 - 6 } & & $C_{n}$ & $C_{a}$ & $C_{n}$ & $C_{a}$ \\
\hline Upper limb & Crawl S & & & & \\
& $\begin{array}{l}\text { Crawl I } \\
\text { Breaststroke } \\
\text { Butterfly } \\
\end{array}$ & 0.67 & 0.20 & 2.95 & 1.61 \\
Backstroke & & & & \\
\hline Lower limb & $\begin{array}{l}\text { Crawl } \\
\text { Backstroke } \\
\end{array}$ & 0.53 & 0.26 & 1.07 & 0.01 \\
& Butterfly & & & & \\
\cline { 2 - 6 } & Breaststroke & 1.88 & 0.50 & 2.30 & 2.38 \\
\hline
\end{tabular}

where $n, M_{\text {sim }}, M_{\text {exp }}, M_{\max }$ and $M_{\min }$ represent the number of time series data for one cycle, simulated value, experimental value, and the maximum and minimum of the experimental values, respectively. That is, the precision, $P$, is the averaged absolute value of the difference between the experimental and simulated values which is divided by the range of the experimental values. The results of comparison between the values of precision, $P$, calculated with the un-unified coefficients in Table 1 and the unified ones in Table 2 are shown in Table 3. From this table, it can be found that the precision did not become worse by the unification of the coefficients for the upper limb. Also for the lower limb, the decline of the precision was 0.03 at the maximum, which could be regarded as sufficiently small. Therefore, it can be concluded that the unification of the coefficients was performed without a significant decrease in the precision.

In order to examine the identified coefficients, they were compared with those in a steady condition. For this purpose, $F_{x}$ and the bending moments at the joints in the steady condition were measured in the situation where the robot arm was fixed in the steady flow, as shown in Fig. 9. The coefficients were then identified as the same as the experiment of the swimming motion. From these results, it was found that $C_{n}$ of the right and left replicas in the steady condition became 0.76 and 0.54 , respectively, which are close to $C_{n}=0.67$ in Table 2.

On the other hand, $C_{n}$ of the forearm in the steady condition was obtained as 0.53 . This value is not significantly different from $C_{n} \approx 0.7$, which is the general value for a cylinder

Table 3 Comparison of precision, $P$, between simulations with un-unified and unified coefficients

\begin{tabular}{llcc}
\hline & & Un-unified & Unified \\
\hline Upper limb & Crawl S & 0.11 & 0.11 \\
& Crawl I & 0.12 & 0.12 \\
& Breaststroke & 0.06 & 0.06 \\
& Butterfly & 0.10 & 0.10 \\
& Backstroke & 0.10 & 0.10 \\
\hline Lower limb & Crawl & 0.14 & 0.17 \\
& Backstroke & 0.08 & 0.09 \\
& Butterfly & 0.14 & 0.17 \\
\cline { 2 - 4 } & Breaststroke & 0.06 & - \\
\hline Average & & 0.10 & 0.11 \\
\hline
\end{tabular}




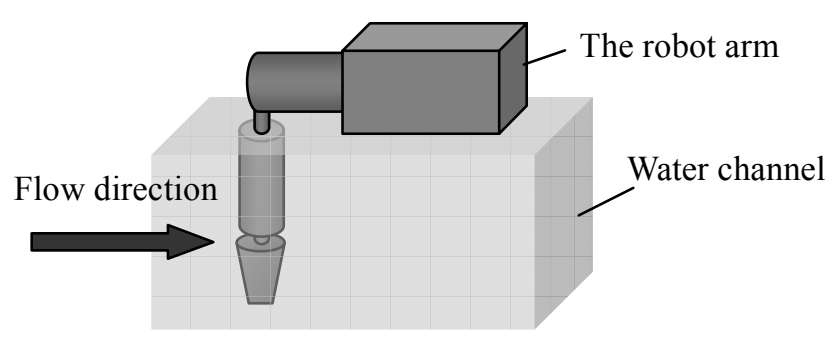

Fig. 9 Experimental setting to measure steady fluid force

with the same slenderness in the steady condition. However, the value of 2.95 during the swimming motion shown in Table 2 is 4 to 6 times larger than 0.5 to 0.7 . The possible reason for this is that the flow around the arm had stronger unsteadiness than that around the hand. The hand basically moves relatively simply in the water, as 'pushing' the water. However, the arm motion is very complicated since many degrees-of-freedom are intricately intertwined. This may enhance the unsteadiness of the flow. It was generally believed that, in swimming, the fluid force acting on the hand mainly contributes to the thrust, while the effect of the arm is small. However, from the present results, it is suggested that the contribution of the arm to the thrust may be larger than previously considered. It is a future task to clarify the reason why such a large fluid force is generated in the unsteady motion of the arm in swimming.

With respect to $C_{a}$, it varied widely due to the upper/lower limb and the stroke, especially for the arm. As described in $\S 3.1, C_{a}$ in the case of a two-dimensional ideal fluid is 1 , which is largely different from the values of Table 2 . It is a future task to clarify the reason for this discrepancy.

\subsection{Simulation using identified fluid force coefficients}

In this section, the results of comparison between the experiment and the simulation using the fluid force coefficients identified in the previous section are shown. The simulation method was the same as the one described in $\S 3.3$. First, the simulation results of the I-shape stroke are described. The swimming motion is shown in Fig. 10. The simulated

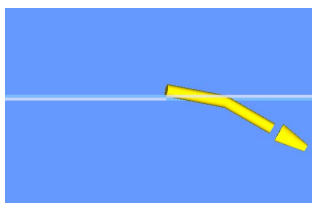

(a) $t=0.0$

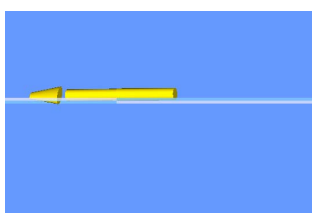

(e) $t=0.4$

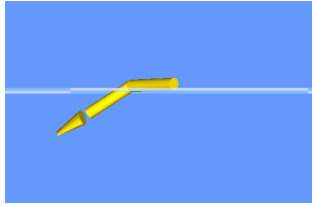

(i) $t=0.8$

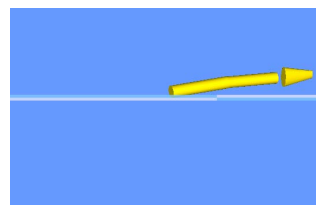

(b) $t=0.1$

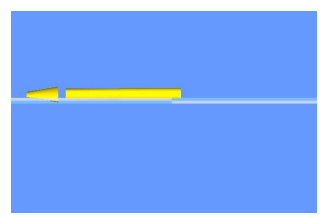

(f) $t=0.5$

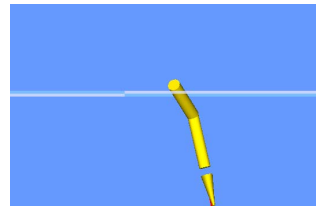

(j) $t=0.9$

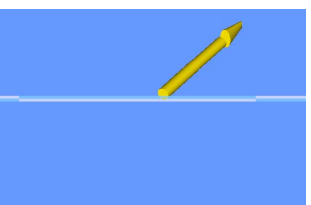

(c) $t=0.2$

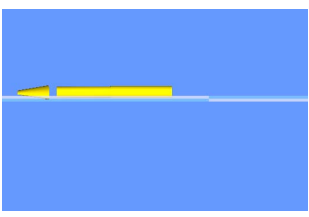

(g) $t=0.6$

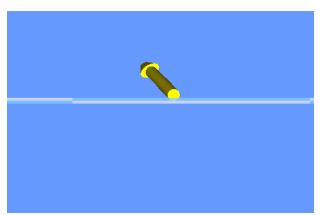

(d) $t=0.3$

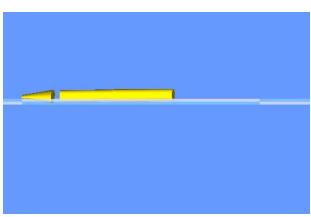

(h) $t=0.7$

Fig. 10 Movement of robot arm in crawl-I upper limb 


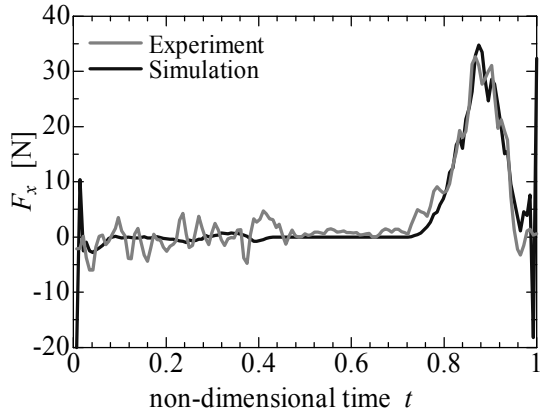

(i) $F_{x}$

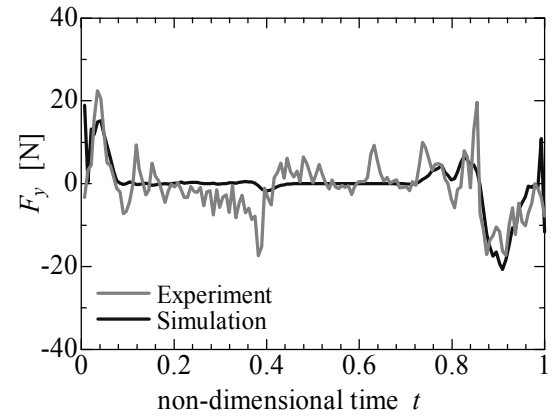

(ii) $F_{y}$

(a) Force acting on the whole robot arm

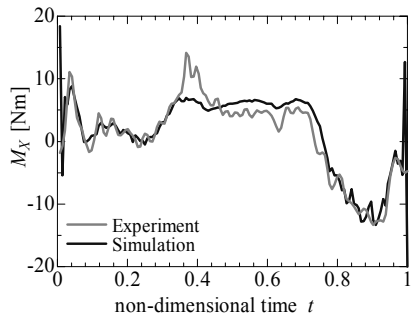

(i) $M_{X}$

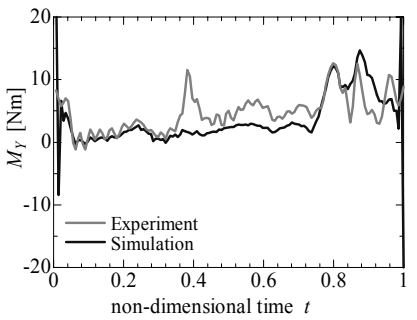

(ii) $M_{Y}$

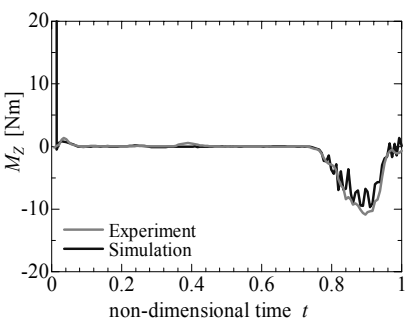

(iii) $M_{Z}$

(b) Moment acting on the upper arm's sensor

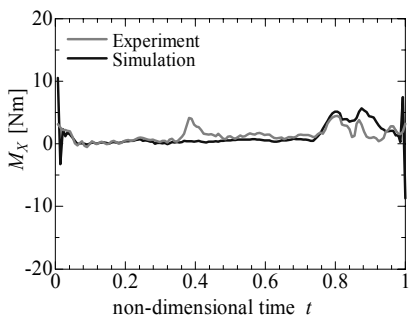

(i) $M_{X}$

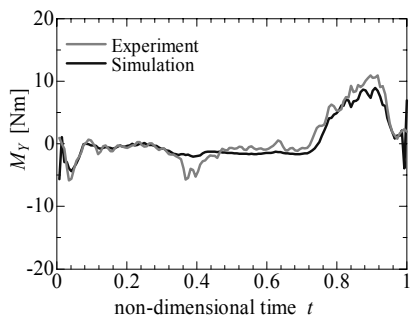

(ii) $M_{Y}$

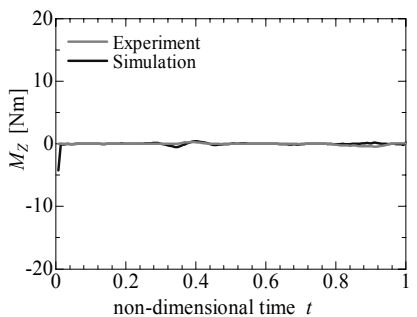

(iii) $M_{Z}$

(c) Moment acting on the forearm's sensor

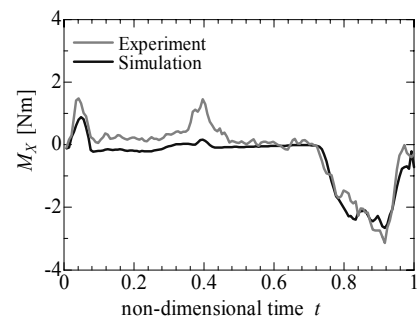

(i) $M_{X}$

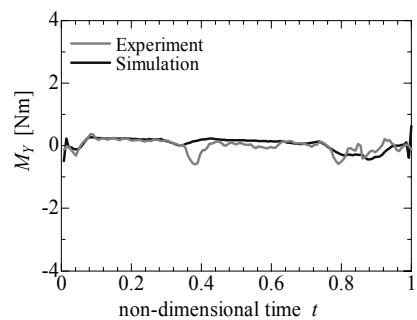

(ii) $M_{Y}$

(d) Moment acting on the hand's sensor

Fig. 11 Comparison between experiment and simulation results in the crawl I upper limb

and experimental results are shown in Fig. 11. Three forces measured by the dynamometer, bending moments at the shoulder, elbow and wrist are shown in Fig. 11(a), (b), (c) and (d), respectively. The abscissa, $t$, is the nondimensional time by which one cycle becomes 1 . The gray and black lines represent the experimental and simulated results, respectively. The timing $t=0.4$ corresponds to the water entry, $t=0.8 \sim 0.9$ the pull motion, $t=0.9 \sim 1.0$ the push motion, and $t=1.0$ the finish. It was found that the simulated results were sufficiently consistent with the experimental results during the period that the arm is underwater. At the timing of the water entry $(t=0.4)$, the error became larger. The reason for this is that the motion of the robot arm at the entry became somewhat unnatural due to mechanical limitation. The simulated results largely deviated from the experimental ones at $t=0$ and 1 . 


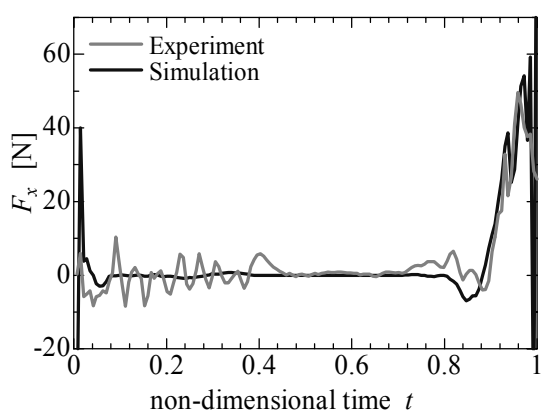

(i) Crawl S (upper limb)

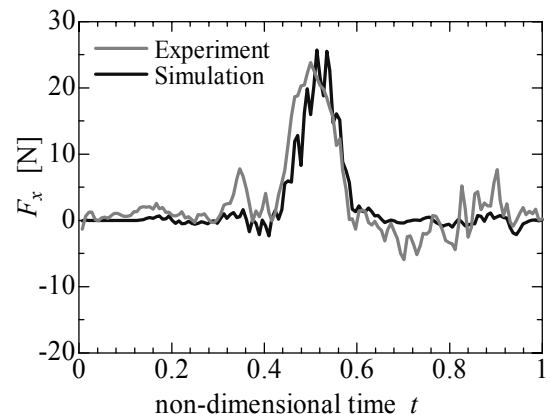

(ii) Butterfly (upper limb)

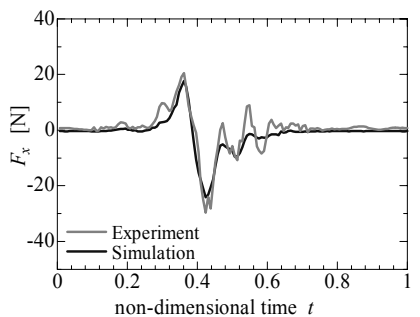

(iii) Breast stroke (upper limb)

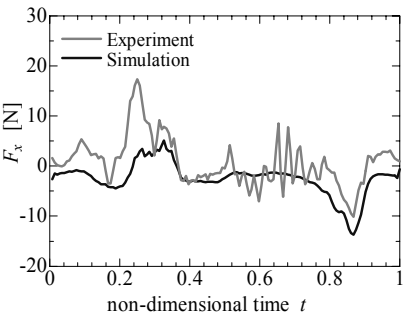

(iv) Backstroke (upper limb)

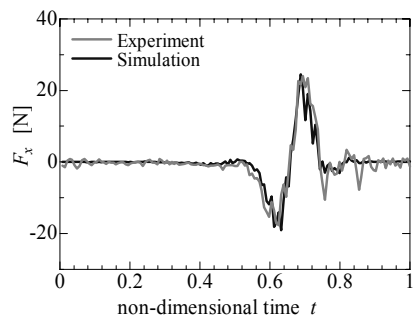

(v) Breaststroke (lower limb)

(a) Dynamometer's Fx

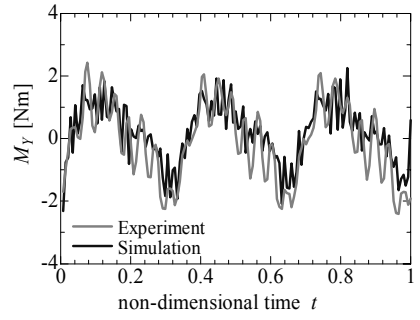

(i) Crawl (lower limb)

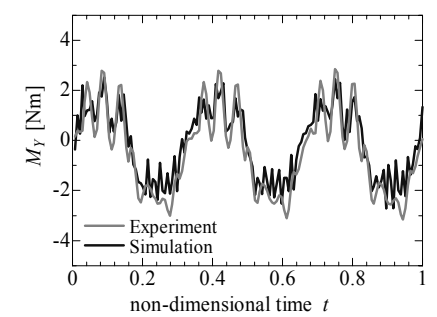

(ii) Backstroke (lower limb)

(b) Shoulder's $M_{Y}$

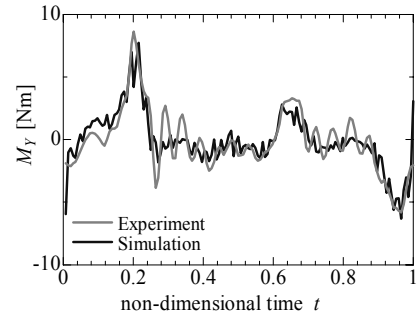

(iii) Butterfly (lower limb)

Fig. 12 Comparison of force and moment between experiment and simulation in each swimming motion

The reason for this is that the motion became unnatural as a whole when the joint angles were interpolated from $t=1$ to $t=0$ in the simulation. The improvement of the simulation for this is a future task.

The results of the simulation and experiment in which the fluid force appeared most remarkably in each stroke are shown in Fig. 12. The results of $F_{x}$ measured by the dynamometer for the S-shaped crawl, butterfly upper limb, breaststroke upper limb, backstroke upper limb and breaststroke lower limb are shown in Fig. 12(a). From the comparison between the results of the simulation and experiment, it was found that the thrust generated by the pull and push motions at $t=0.8 \sim 1.0$ for the S-shaped crawl and at $t$ $=0.5$ for the butterfly upper limb was reproduced well in the simulation. The reason for the discrepancy between the results of the simulation and experiment at $t=0$ and 1 is the error of the interpolation from $t=1$ to $t=0$, the same as in the I-shaped crawl. For the other strokes, the influence of the error due to the interpolation did not become remarkable because of the absence of large motions at $t=0$ and 1 . For the breaststroke upper limb, the thrust generated by the pull motion at $t=0.3$, as well as the negative thrust by the recovery motion at $t=0.4$, were reproduced well. For the backstroke, however, the discrepancy was larger than those for the other strokes, especially at the pull motion $(t=0.2 \sim 0.3)$. A possible reason for this is that the motion of the robot arm became unnatural due to mechanical limitation, and therefore the stroke became very close to the water surface. For the 
breaststroke lower limb, the negative thrust by the pull motion of the leg at $t=0.6$ and the thrust by the kick at $t=0.7$ were reproduced very well. The results of the bending moment at the hip joint, $M_{Y}$, for the flutter kick of the crawl and backstroke as well as the butterfly lower limb are shown in Fig. 12(b). For the flutter kick of the crawl and backstroke, the fluid force generated by the three kicks in one cycle was reproduced well. For the butterfly lower limb, the weak kick at $t=0.6$ and the strong kick at $\mathrm{t}=0.9 \sim 1.0$ were represented well. From all the above results, it can be said that the simulation results sufficiently reproduced the experimental results for all strokes unless the motion of the robot arm was unnatural. Therefore, it was confirmed that the fluid forces acting on the four limbs during swimming could be sufficiently reproduced by the present fluid force model.

\section{Conclusion}

In this paper, the fluid force model was first formulated, and a simulation model which reproduces the experiment to measure the unsteady fluid force using an underwater robot arm, was developed. The fluid force coefficients for all strokes were then identified based on the comparison between the results of the simulation and the experiment. By unifying the coefficients for each shape, a versatile fluid force model, which depends not on stroke but only on shape, was determined. The validity of this fluid force model was confirmed since the simulation results using the fluid force model sufficiently reproduced the experimental results for all cases, although some discrepancies were seen for the motion close to the water surface or at the start or end of a cycle. Various mechanical analyses for the various strokes will be possible by this model. The limitations of the present model include that it is based on an experiment in which the Reynolds number is not consistent with that of actual swimming and that the shape fidelity of the physical limb models are not complete, as well as the discrepancy for the motion close to the water surface as described in this paper. In addition to these, clarification of the unsteady fluid forces acting on the body trunk is also an important task since the fluid forces acting on the trunk will be as large as those on the limbs in swimming. These problems should be solved by certain methods in the future.

\section{References}

(1) Takahashi, A., Nakashima, M., Measurement and Modeling of Unsteady Fluid Force Acting on Limbs in Swimming Using an Underwater Robot Arm, Transactions of the Japan Society of Mechanical Engineers, Series B, Vol.75, No.750 (2009), pp.284-293.

(2) Schleihauf, R.E., A Hydrodynamic Analysis Of Swimming Propulsion, Swimming III, (1979), pp.70-109, University Park Press.

(3) Berger, M.A.M., de Groot, G. Hollander A.P., Hydrodynamic Drag and Lift Forces on Human Hand/Arm Models, Journal of Biomechanics, Vol.28, No. 2 (1995), pp.125-133.

(4) Ito, S., Okuno, K., A Fluid Dynamical Consideration for Arm Stroke in Swimming, Biomechanics and Medicine in Swimming IX, (2003), pp.39-44.

(5) Nakashima, M., Satou, K., Miura Y., Development of Swimming Human Simulation Model Considering Rigid Body Dynamics and Unsteady Fluid Force for Whole Body, Journal of Fluid Science and Technology, Vol.2, No.1 (2007), pp.56-67.

(6) Nakashima, M., Mechanical Study of Standard Six Beat Front Crawl Swimming by Using Swimming Human Simulation Model, Journal of Fluid Science and Technology, Vol.2, No.1 (2007), pp.290-301.

(7) SWUM developer team, "SWUM home page", SWUM website, (online), available from <http://www.swum.org/>, (accessed 2009-9-22)

(8) AIST. "AIST Measurement Database of Japanese Body Size Data (in Japanese) 1991-92”. AIST. (online), available from $<$ http://riodb.ibase.aist.go.jp/dhbodydb/91-92/>, (accessed 2009-9-22) 
(9) Nakashima, M., Development of the Swimming Human Simulation Software "Swumsuit", Journal of The Japan Society For Simulation Technology, Vol.26, No.1 (2007), pp.58-66.

(10) Nakashima, M. "Swimming Human Simulation Software "Swumsuit"”. Swimming Human Simulation Software "Swumsuit". (online), available from $<$ http://www.swum.org/swumsuit/>, (accessed 2009-9-22) 